\title{
Optimizing diffusion in multiplexes by maximizing layer dissimilarity
}

\author{
Alfredo B. Serrano, ${ }^{1}$ Jesús Gómez-Gardeñes, ${ }^{2,3}$ and Roberto F. S. Andrade ${ }^{1}$ \\ ${ }^{1}$ Instituto de Física, Universidade Federal da Bahia, 40210-210 Salvador, Brazil \\ ${ }^{2}$ Department of Condensed Matter Physics, University of Zaragoza, 50009 Zaragoza, Spain \\ ${ }^{3}$ Institute for Biocomputation and Physics of Complex Systems, University of Zaragoza, 50018 Zaragoza, Spain
}

(Received 27 January 2017; published 17 May 2017)

\begin{abstract}
Diffusion in a multiplex depends on the specific link distribution between the nodes in each layer, but also on the set of the intralayer and interlayer diffusion coefficients. In this work we investigate, in a quantitative way, the efficiency of multiplex diffusion as a function of the topological similarity among multiplex layers. This similarity is measured by the distance between layers, taken among the pairs of layers. Results are presented for a simple two-layer multiplex, where one of the layers is held fixed, while the other one can be rewired in a controlled way in order to increase or decrease the interlayer distance. The results indicate that, for fixed values of all intra- and interlayer diffusion coefficients, a large interlayer distance generally enhances the global multiplex diffusion, providing a topological mechanism to control the global diffusive process. For some sets of networks, we develop an algorithm to identify the most sensitive nodes in the rewirable layer, so that changes in a small set of connections produce a drastic enhancement of the global diffusion of the whole multiplex system.
\end{abstract}

DOI: 10.1103/PhysRevE.95.052312

\section{INTRODUCTION}

Multilayer networks have been the focus of intense research in recent times [1,2]. Such interest arises mainly from the necessity of exploring new emergent properties in networks whose backbone is formed by different types of connections [3-5]. Many aspects of network theory have been recently revisited under the paradigm of multilayer systems. These features cover the stability of technological interdependent networks that has caused a catastrophic breakdown of energy distribution [6], mathematical aspects related to their spectral properties [7,8], and critical phenomena [9].

During recent years, most of the attention has been devoted to a particular class of multilayer networks called multiplexes. A multiplex is a multilayer structure formed by $M$ layers, each of them being itself a network [5,10,11] and containing exactly the same quantity, $N$, of nodes. This way, we can represent systems in which a set of $N$ nodes can be connected through links of $M$ types. Such a feature can be effectively observed in a series of actual complex systems, e.g., a set of airports that are connected by different airlines, each one with its own set of flights connecting a common pool of destinations [12,13]. The same is observed for a group of individuals that may communicate with each other through different media [14] or use different communication means with the same set of locations [15-17].

To assemble a multiplex (see Fig. 1), we represent each of the $N$ entities (airports, individuals, locations, etc.) in each of the $M$ layers, so that for each entity there are $M$ nodes (one per layer) that represent it. Each of the $N$ nodes in a layer is directly connected to its $M-1$ counterparts in the other layers as they represent the same entity while the rest of the connections are established within the layer to which they belong.

The particular form of multiplexes and its ubiquity as the backbone of real complex systems has motivated the development of a mathematical framework for their treatment [11]. This has helped the analysis of the emergence of collective behavior such as percolation [18-20], epidemics [21-26], coordination [27,28], cooperation [29-32], and synchronization [33-36], among others [37]. In many cases, these studies have shed light on the new physical phenomena that the coupling between the interaction layers of the multiplexes induces to the collective behavior of such systems [38].

A general issue related to multiplex systems is the understanding of diffusive processes on such a structure, and, particularly, its relation to the diffusive properties of each interaction when considered independently [39]. Multiplex diffusion depends on diffusion within each individual layer, $\alpha=1,2, \ldots, M$, but also on the interlayer diffusion coefficients $D_{\alpha, \beta}$. If these are all set to zero, multiplex diffusion is restricted to each specific layer, depending only on the specific link distribution in that layer and on the intralayer diffusion coefficients. In the other extreme, the full potential of the multiplex is reached when all $D_{\alpha, \beta} \neq 0$, which allows a direct connection between any pair of layers. Therefore, it is quite a difficult task to predict, in a general way, how a global multiplex diffusive process depends on each of the individual intralayer counterparts.

In this work, we propose to relate the efficiency of the global multiplex diffusion to a quantitative measure for the difference between the topological structure of any pair of layers in the multiplex. In a single layer $\alpha$, the diffusion efficiency, which depends on the intralayer diffusion coefficient $D_{\alpha}$ and on the network topology, is usually expressed in terms of the smallest nonzero eigenvalue $\lambda_{2}^{\alpha}$ of the corresponding Laplacian matrix $L_{\alpha}$ [40]. Similarly, multiplex diffusion is expressed by $\Lambda_{2}$, the smallest nonzero eigenvalue of the supra-Laplacian matrix, as will be detailed in the next section. Thus, we investigate how $\Lambda_{2}$ depends on the dissimilarity between layers, measured through the network distance introduced in [41]. In particular, for a multiplex, we can evaluate $M(M-1) / 2$ values $\delta(\alpha, \beta)$ corresponding to the distance between a pair of layers $\alpha$ and $\beta$. For the sake of a clearer presentation of our results, we restrict our analysis to the simplest situation of an $M=2$ multiplex to follow in a close way the relation between $\Lambda_{2}$ and the layer distance $\delta$ while proceeding with a controlled rewiring of one of the networks.

The rest of this work is organized as follows: In Sec. II, we show how to describe a diffusion process in a multiplex by 


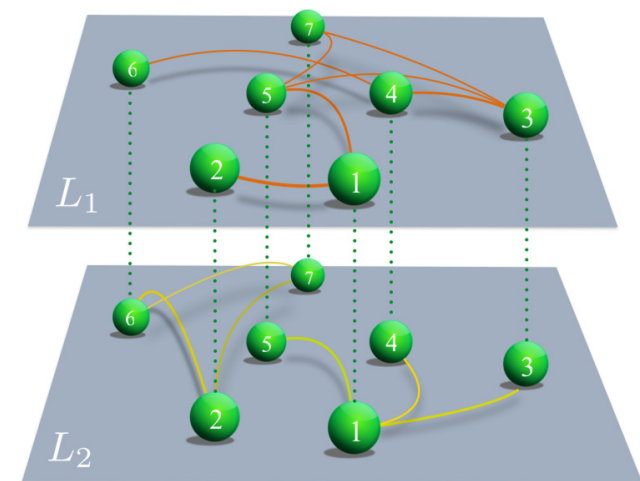

FIG. 1. Illustration of a simple multiplex network composed of $M=2$ layers and $N=7$ nodes. Note that each node is represented once in each of the two interaction layers, and that an interlayer connection (dotted lines) is established between its two representations.

means of the supra-Laplacian matrix. Section III discusses the method used to rewire a multiplex layer, providing a physical interpretation to this process and its relation to $\delta(\alpha, \beta)$. Section IV presents our results for multiplexes constituted by layers belonging to well-known network classes [42-46] such as Erdös-Renyi (ER) [47,48], Barabási-Albert (BA) [49], and Watts-Strogatz (WS) [50] networks. Finally, Sec. V closes the paper with conclusions and remarks on future developments.

\section{DIFFUSION IN MULTIPLEXES}

Diffusion is a well-known irreversible physical process by which particles move from one part of the system to another with a corresponding increase of the entropy. The transport of particles occurs in the inverse direction of the concentration gradient, i.e., from a high concentration to one of lower concentration. Analogous versions of diffusion processes also occur in complex networks, related to information spread in social networks, flows of people in transportation systems, or epidemic propagation of diseases. As the network topology has a great influence on spreading processes, it becomes quite important to understand the relation between them in order to control diffusion.

Consider a network characterized by the set $V$ of $N$ vertices (or nodes), and the set $E$ of edges. It can be represented by its adjacency matrix $A$ with elements $a_{i j}$. For the sake of simplicity, we assume the network is undirected, although the formalism described here can be adapted for directed networks. If we consider a multiplex with $M$ layers, the set $V$ is the same for all layers, but, in each of them, the representation of node $i$ receives a different label $i+(\alpha-1) N$, with $\alpha=1,2, \ldots, M$. The edges in each layer are described by different sets $E_{\alpha}, \alpha=1,2, \ldots, M$, so that the multiplex adjacency matrix $\mathcal{A}$ is assembled by the $M$ corresponding adjacency matrices $A_{\alpha}$, with the further addition of $N M(M-1) / 2$ links between each node $i$ and its counterparts in the $M$ layers. For the simplest case $M=2, \mathcal{A}$ has the following structure:

$$
\mathcal{A}=\left(\begin{array}{cc}
A_{1} & 0 \\
0 & A_{2}
\end{array}\right)+\left(\begin{array}{ll}
0 & I \\
I & 0
\end{array}\right),
$$

where $I$ represents the $N \times N$ identity matrix describing the interlayer connections between the representations of a node in each of the $M=2$ layers.
To describe the linear diffusive process on the multiplex, we follow the framework introduced in Ref. [39]. Let the vector $\mathbf{x}$ of components $\left(x_{\alpha}\right)_{i}$ represent the state of the diffusing substance in the node $i+(\alpha-1) N$. By assuming that the state in each node changes only due to differences in the values of $\left(x_{\alpha}\right)_{i}$ with respect to that in the nodes to which it is directly connected, the time evolution of $\mathbf{x}$ is controlled by the following equation:

$$
\begin{aligned}
\frac{d\left(x_{\alpha}\right)_{i}}{d t}= & D_{\alpha} \sum_{j=1}^{N}\left(a_{\alpha}\right)_{i j}\left[\left(x_{\alpha}\right)_{j}-\left(x_{\alpha}\right)_{i}\right] \\
& +\sum_{\beta=1}^{M} D_{\alpha \beta}\left[\left(x_{\beta}\right)_{i}-\left(x_{\alpha}\right)_{i}\right]
\end{aligned}
$$

where $D_{\alpha}$ and $D_{\alpha \beta}$ represent, respectively, the intralayer diffusion coefficient within each layer $\alpha$ and the interlayer diffusion coefficient between the identical nodes in the layers $\alpha$ and $\beta$. To describe a more general process in which the intralayer diffusion is not homogeneous, it is necessary to replace each adjacency matrix $A_{\alpha}$ by the corresponding weighted matrix $W_{\alpha}$ [51], with elements $\left(w_{\alpha}\right)_{i, j}$ representing the strength of the interaction between each pair of nodes.

In the case of a single layer $(M=1)$, Eq. (2) reduces to the usual network diffusion equation $\dot{x}=-L x$, where $L$ is the Laplacian matrix with elements $L_{i j}=k_{i} \delta_{i j}-a_{i j}, k_{i}$ indicating the degree of the node $i . L$ is a square and symmetric matrix, with real eigenvalues $\lambda_{i}$, and at least one vanishing eigenvalue $\lambda_{1}=0$. The relaxation time $\tau$ of the diffusive process is controlled by the smallest nonzero eigenvalue, which we will assume to be $\lambda_{2}=1 / \tau$.

For a general multiplex with $M>1$ layers, Eq. (2) can also be written in matrix form with the help of the $M N \times M N$ supra-Laplacian matrix defined as [39]

$$
\mathcal{L}=\mathcal{L}_{0}+\mathcal{L}_{s},
$$

where $\mathcal{L}_{0}$ is a block-diagonal matrix where each block $\alpha$ is the usual Laplacian matrix corresponding to the $\alpha$ th layer, while $\mathcal{L}_{s}$ describes the interaction between the corresponding nodes on different layers $\alpha$ and $\beta$. The off-diagonal blocks of $\mathcal{L}_{s}$ correspond to the adjacency matrix with the negative sign multiplied by the corresponding diffusion coefficients $D_{\alpha \beta}$. Given that interlayer connections are only allowed between the same node in different layers, the off-diagonal blocks consist of $N \times N$ diagonal matrices. The diagonal blocks of $\mathcal{L}_{s}$ are also diagonal matrices, the elements $\left(\mathcal{L}_{s}\right)_{i i}$ of which are the negative sum of all elements in the row $i$ of $\mathcal{L}_{s}$. Therefore, when all interlayer diffusion coefficients are equal, i.e., $D_{\alpha \beta}=D_{x}$, we obtain

$$
\begin{aligned}
\mathcal{L} & =\left(\begin{array}{cccc}
L_{1} & 0 & \cdots & 0 \\
0 & L_{2} & \cdots & 0 \\
\vdots & \vdots & \ddots & \vdots \\
0 & 0 & \cdots & L_{M}
\end{array}\right) \\
& +D_{x}\left(\begin{array}{cccc}
(M-1) I & -I & \cdots & -I \\
-I & (M-1) I & \cdots & -I \\
\vdots & \vdots & \ddots & \vdots \\
-I & -I & \cdots & (M-1) I
\end{array}\right) .
\end{aligned}
$$


By evaluating $\Lambda_{2}$, the smallest nonzero eigenvalue of $\mathcal{L}$ in (4), we characterize the essential features of multiplex diffusion.

Since in this work we restrict our investigation to $M=2$ multiplexes, we can use the results derived in [39] for the dependency of the smallest nonzero eigenvalue of $\mathcal{L}$ on the interlayer diffusion coefficient. To this purpose, we assume that $D_{1}=D_{2}=1$ and look for the function $\Lambda_{2}=\Lambda_{2}\left(D_{x}\right)$. In [39], exact analytical results for two extreme cases have been obtained:

(i) When the interlayer coupling is small, $D_{x} \ll 1$, $\Lambda_{2}\left(D_{x}\right)=2 D_{x}$.

(ii) For large interlayer coupling, $D_{x} \rightarrow \infty, \Lambda_{2}\left(D_{x}\right) \sim$ $\bar{\lambda} / 2$, where $\bar{\lambda}$ is the first nonzero eigenvalue of the Laplacian matrix $\bar{L}=\left(L_{1}+L_{2}\right) / 2$ corresponding to the superposition of the two layers.

It is interesting to note that, given the smallest nonzero eigenvalues of each layer, $\lambda_{2}^{\alpha}$ and $\lambda_{2}^{\beta}$, in the strong-coupling regime $\Lambda_{2} \geqslant \min \left(\lambda_{2}^{\alpha}, \lambda_{2}^{\beta}\right)$ is always satisfied, meaning that diffusion in the multiplex is always faster than in the slowest layer. However, superdiffusion occurs when $\Lambda_{2} \geqslant$ $\max \left(\lambda_{2}^{\alpha}, \lambda_{2}^{\beta}\right)$. In [39] it was shown that superdiffusion is possible for certain combinations of network topologies. Although no rigorous conditions for its emergence were derived, it was hypothesized that superdiffusion should be fostered when the number of redundant links in both layers is minimum. This argument serves us in the following section to design multiplexes for which the diffusion rate is maximized.

For intermediate values of $D_{x}$, we must conduct a numerical evaluation of $\Lambda_{2}$. Therefore, in Sec. IV we discuss our results based on graphs of $\Lambda_{2}$ as a function of $D_{x}$, much as was done in [39].

\section{CONTROLLED MULTIPLEX REWIRING}

\section{A. Network distance}

Given any two networks $\alpha$ and $\beta$ with the same number of nodes $N$, it is possible to define a distance $\delta$ between them by the expression [41]

$$
\delta^{2}=\frac{1}{N(N-1)} \sum_{i, j=1}^{N}\left[\frac{\left(\widehat{V_{\alpha}}\right)_{i, j}}{\mathfrak{D}_{\alpha}}-\frac{\left(\widehat{V_{\beta}}\right)_{i, j}}{\mathfrak{D}_{\beta}}\right]^{2} .
$$

Here, $\mathfrak{D}_{\alpha}$ and $\mathfrak{D}_{\beta}$ indicate the diameter of each network, while $\widehat{V_{\alpha}}$ and $\widehat{V_{\beta}}$ are the corresponding neighborhood matrices [52], the elements of which indicate the length of the shortest path between the corresponding pairs of nodes in the network.

Let us remark that the $N$ nodes in a network may be numbered $N$ ! different ways, which do not affect the network topology. If the nodes are pure mathematical entities, or if they represent indistinguishable physical entities, they can be freely renumbered, with no further consequences. If the nodes have identifiable attributes they can also be renumbered, but in such cases it is necessary to keep track of the attributes associated with each node when the network is renumbered.

It is easy to see that the results provided by Eq. (5) depend on the specific node numbering. Thus, to consistently use $\delta$ as a measure of the topological difference between two networks $\alpha$ and $\beta$ where the nodes can be freely renumbered, it becomes necessary to obtain $\Delta(\alpha, \beta) \equiv \delta_{\min }$, i.e., the minimum value of $\delta$ taken over all possible node numberings of one of the networks, keeping fixed the numbering of the other one. Unless $N$ is very small, it becomes prohibitive to compute all $N$ ! values of $\delta$, which requires an alternative procedure to obtain a good estimation for $\Delta(\alpha, \beta)$. This can be achieved by a Monte Carlo (MC) procedure based on the METROPOLIS algorithm [53] to minimize $\delta$ using a random sequence of pairwise renumbering steps. With the exception of a small number of upward jumps allowed by the MC algorithm, $\delta$ decreases monotonically as $s$ increases, where $s$ indicates the number of undertaken MC steps [41].

\section{B. Monte Carlo multiplex rewiring}

In the current study, our focus is understanding diffusion on $M=2$ multiplexes with $N$ nodes as a function of differences in the layer topologies. We state that a multiplex is formed (or assembled) when a direct interlayer connection is inserted between every node with the same label (number, name, subject, etc.) in two hitherto isolated networks, say $\alpha$ and $\beta$. If $\alpha$ and $\beta$ are arbitrary networks, i.e., if the nodes are all equivalent and have no specific meaning, they can be independently numbered in $N$ ! ways in each of the networks. At the same time, there exist $N$ ! ways to assemble a multiplex based on $\alpha$ and $\beta$, which can be accomplished, e.g., by holding the node numbering in $\alpha$ constant, spanning the $N$ ! different $\beta$ numbering and, for each of them, inserting the direct interlayer connections. If the multiplex is formed by two networks where the nodes in each of them have some precise meaning, there is only one possible way to insert interlayer connections between the nodes.

The straightforward way to set a dependency between diffusion and topology difference is to evaluate $\Lambda_{2}\left(D_{x} ; \alpha, \beta\right)$ and $\delta(\alpha, \beta)$ for multiplexes that are constituted by chosen networks $\alpha$ and $\beta$. If they are arbitrary, we can freely use the MC approach described before to obtain a significative sample of the $N$ ! multiplexes and the corresponding dependence between $\Lambda_{2}$ and $\delta$. If we investigate multiplexes with identifiable nodes, renumbering nodes in $\beta$ by the MC procedure is also possible, but the interpretation of the process is different from that provided in [41]. As it is not possible to renumber the nodes of only one layer, the process of switching the labels of two nodes in $\beta$ is equivalent to switching all connections they have in $\beta$, giving rise to a new connection landscape in that layer. Note that this interpretation is also valid when we work with two arbitrary networks.

The use of the MC algorithm to identify $M=2$ multiplexes where global diffusion proceeds at a faster or slower pace is the key contribution of this work. Each MC step allowing a change in $\beta$ can be represented by three diagrams in Fig. 2:

(i) A pair of nodes that will switch labels in $\beta$ is chosen.

(ii) Switching labels does not make the nodes in $\beta$ loose their original identification with nodes in $\alpha$, which is indicated by tilted dashed lines between the two layers.

(iii) Relabeled nodes are moved back to their original positions in $\beta$, changing the graphical representation of the layer $\beta$ but not its topology.

Because of that, the intralayer diffusion on $\beta$ is not affected as $\lambda_{2}^{\beta}$ is not affected by node renumbering. However, this 

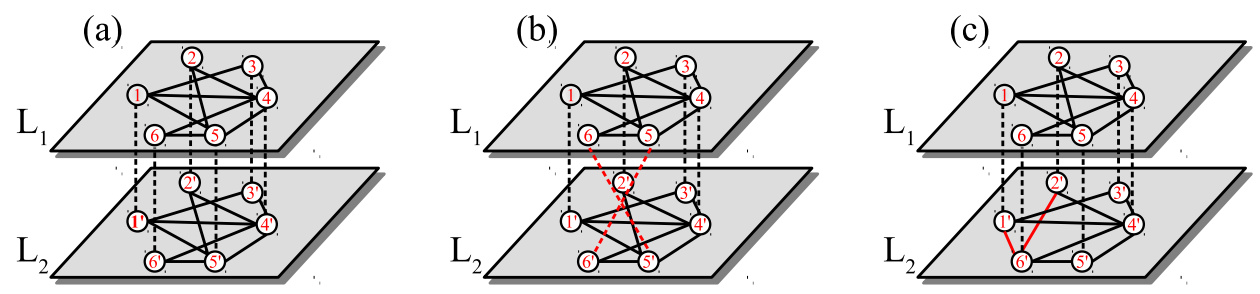

FIG. 2. Illustration of the rewiring process in the layer $\mathbf{L}_{2}$ associated to switching the labels of nodes 5 and 6 . In (a), the two layers are identical. In (b), the labels $5^{\prime}$ and $6^{\prime}$ have been switched, but they are still connected to nodes 5 and 6 by tilted lines. In (c), the new nodes $5^{\prime}$ and $6^{\prime}$ are moved back to the positions vertically below the nodes 5 and 6 , resulting in a displacement of the connections in $\mathbf{L}_{\mathbf{2}}$.

procedure affects the general topological structure of the multiplex, which may impact the global multiplex diffusion. The MC procedure can be set to accept changes that either increase or decrease $\delta$ by rewiring $\beta$.

The procedure starts by tuning the MC algorithm to increase or decrease $\delta$, and selecting an $M=2$ multiplex assembled by the layers $\alpha$ and $\beta \equiv \beta(s=0)$. Next, the MC algorithm is sequentially applied, and if $s$ indicates the number of MC steps in the process, we obtain a series of values $\delta(s)=\delta(\alpha, \beta(s))$ and the corresponding series of multiplexes $\beta(s)$. Once $N$ is finite, the procedure will eventually lead $\delta(s)$ to reach a plateau where it shows just small fluctuations. The highest (smallest) attained value for $\delta(s)$ is the best estimate for $\bar{\Delta}(\alpha, \beta) \equiv \delta_{\max }(s)\left[\Delta(\alpha, \beta) \equiv \delta_{\min }(s)\right]$. This process is coupled to the evaluation of $\Lambda_{2}\left(D_{x} ; s\right)$ at a conveniently chosen interval of MC steps, providing a clear picture of the relation between multiplex diffusion and topology. One particularly useful strategy is to choose $\beta(s=0)=\alpha$, so that $\delta(0)=0$.

\section{Optimized rewiring}

As described above, the rewiring procedure based on the MC algorithm will eventually find the optimized $\beta(s)$ for which $\delta(s) \simeq \bar{\Delta}(\alpha, \beta)$, although this may require a large number $S$ of MC steps. Nevertheless, one may question whether it is possible to relabel a limited number $\sigma \ll S$ of specific nodes in such a way that $\delta(\sigma) \lesssim \bar{\Delta}(\alpha, \beta)$. Although it is not possible to provide a simple answer for every choice of $\alpha$ and $\beta$, we were able to devise two procedures that are able to fulfill this task when $\beta(s=0)=\alpha$, provided they satisfy some specific topological properties.

The first procedure is based on the comparison of the neighborhood structures of each node. The $i$ th line of the neighborhood matrix $\widehat{V}$ contains the distance of node $i$ to all other nodes $j \neq i$. Therefore, we define

$$
t_{i, \ell}=\frac{1}{N} \sum_{j=1}^{N}\left[\widehat{V}_{i, j}-\widehat{V}_{\ell, j}\right]^{2}
$$

which is proportional to the difference between the neighborhoods of nodes $i$ and $\ell$. By summing the above expression over $\ell$, we obtain

$$
T_{i}=\frac{1}{N-1} \sum_{\ell=1}^{N} t_{i, \ell},
$$

which expresses the average distance from the node $i$ to all other nodes. Now we order the nodes according to decreasing values of $T_{i}$ and consider the node at the top position, $\hat{i}$, for which $T_{\hat{i}}>T_{i}, \forall i \neq \hat{i}$. This node is somewhat peculiar, as it has the largest topological difference from the other nodes in the network. This hints that, by rewiring its connections, one might cause a large change in the network topology. After selecting $\hat{i}$, which constitutes the step (i) for selecting a pair of nodes to be rewired, we move to step (ii) to find the node $\hat{\ell}$ that has the largest contribution to $T_{\hat{i}}$, i.e., satisfying the condition $t_{\hat{i}, \hat{\ell}}>t_{\hat{i}, \ell}, \forall \ell \neq \hat{\ell}$. Thus, steps (i) and (ii) select the pair $(\hat{i}, \hat{\ell})$ that will have the labels switched.

As in the random rewiring procedure, the optimized rewiring starts with $\delta(s=0)=0$, and the pair $(\hat{i}, \hat{\ell})$ is rewired. To proceed with the procedure and select the second pair of nodes to be rewired, we still implement step (iii): subtract from $T_{\hat{i}}$ the contribution $t_{\hat{i}, \hat{\ell}}$. After that, the optimized rewiring proceeds by successively applying steps (i)-(iii). As we will show in the next section, this procedure is particularly efficient to cause huge changes in the diffusion process by switching only a very small number of pairs of nodes when the initial layers are identical ER networks.

The second procedure follows the same steps indicated above, but the criterion is based on selecting pairs of nodes with the largest and smallest values of node degree. It turns out that this method is more efficient to cause changes in the diffusion processes when the initial layers correspond to identical BA networks.

Results for the behavior of $\delta(s)$ as a function of $s$ for ER and BA multiplexes are shown in Fig. 3. In panel (a), we draw the behavior of $\delta(s)$ as a function of $s$ for a multiplex assembled at $s=0$ by identical $N=500$ ER layers where each pair of nodes is connected with probability $p=0.01$. When $\beta(s)$ is rewired according to the random MC algorithm, $\delta(s)$ increases with $s$ along a sigmoidal function, which seems to have reached a plateau at $\sim 5 \times 10^{6} \mathrm{MC}$ steps. Similar patterns are obtained when we consider a multiplex assembled by identical BA or WS layers. On the other hand, our results also indicate that $\delta(s)$ increases at a much larger pace for the optimized rewiring as compared to the random process for ER networks. This behavior is not reproduced when BA or WS multiplexes are rewired by the optimized procedure.

The results in panel (b) indicate the typical behavior for multiplexes formed by distinct realizations of ER networks. The two curves $\delta_{ \pm}(s)$ evolve from the value $\delta(s=0)$ moving toward $\bar{\Delta}(\alpha, \beta)$ and $\Delta(\alpha, \beta)$ as $s$ increases. The pattern observed for an $N=1000$ BA multiplex in panel (c) is similar, although we notice a change in the asymmetry of $\delta_{+}(s)$ and $\delta_{-}(s)$ with respect to the horizontal line $\delta(s)=\delta(s=0)$. 

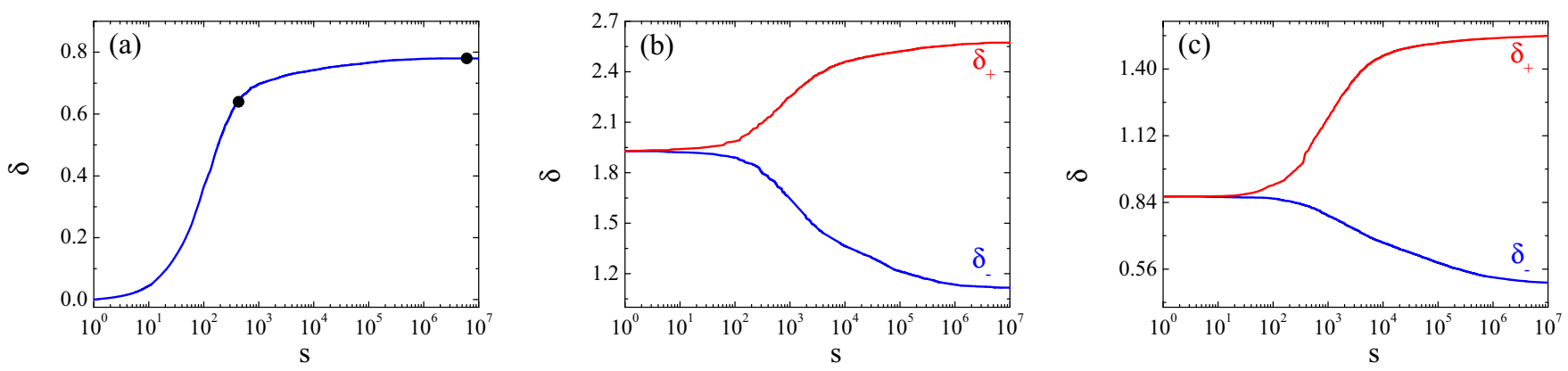

FIG. 3. Typical results for the behavior of $\delta(s)$ as a function of $s$ resulting from the rewiring procedure. (a) Multiplex assembled at $s=0$ by one single $N=1000, p=0.01$ ER network. The solid circles indicate the values $s=434$ and $s=5 \times 10^{6}$. (b) Multiplexes assembled at $s=0$ by two distinct samples of $N=500, p=0.01$ ER networks. (c) Multiplexes assembled at $s=0$ by two distinct samples of $N=1000$ BA networks. In (b) and (c), the two branches indicated by $\delta_{+}$and $\delta_{-}$are obtained by tuning the MC procedure to increase and decrease the value of $\delta(s)$.

These specific forms are typical for results obtained by joining different ER and BA realizations, respectively, and they also seem to be independent of the chosen number of nodes.

\section{RESULTS}

The essential information on global multiplex diffusion is provided by the behavior of $\Lambda_{2}$ as a function of $D_{x}$. If we take into account that $\Lambda_{2}$ can only be numerically evaluated for almost all values of $D_{x}$, its graphical representation becomes the most convenient way to access and analyze the results. Also, to better display the different regions of values of $D_{x}$, it is wise to use logarithmic scales in both axes.

The quantitative discussion of these plots is better perceived when we take into account the expressions for $\Lambda_{2}$ under the conditions $D_{x} \ll 1$ and $D_{x} \gg 1$, as indicated in Sec. II. Therefore, we include in all graphs the tilted line $\Lambda_{2}=2 D_{x}$ as well as a horizontal line at the value $\bar{\lambda}_{2}$, indicating the asymptotic value of $\Lambda_{2}$ in the second limiting conditions. To keep track of the diffusive properties of each individual layer, henceforth identified by the labels $\alpha$ and $\beta$, we add to the graphs two further horizontal lines at the values $\lambda_{2, \alpha}$ and $\lambda_{2, \beta}$. An observed feature in all studied cases is the monotonic increase of $\Lambda_{2}$ with $D_{x}$. Therefore, the obtained pattern shows how $\Lambda_{2}$ stays under the influence of each regime and how it behaves in the intermediary region.

This is exemplarily illustrated in Fig. 4, where panel (a) shows $\Lambda_{2}\left(D_{x} ; s\right) \times D_{x}$ for two multiplexes assembled by $N=500, p=0.01$ ER networks, with the inclusion of the additional curves described above. For both multiplexes, we consider the same layer $\alpha$ but different layers $\beta$. In the first case, the layer $\beta(s=434)$ was obtained by rewiring $s=434$ pairs of nodes from $\alpha$ according to the random procedure discussed in Sec. III [see the first dot in Fig. 3(a)]. For the second multiplex, the second layer is $\beta\left(s=10^{7}\right)$, obtained in a similar way after rewiring a much larger number of randomly chosen pairs of nodes.

In the small $D_{x}$ region, both $\Lambda_{2}\left(D_{x} ; s=434\right)$ and $\Lambda_{2}\left(D_{x} ; s=10^{7}\right)$ collapse with the exact asymptotic result $\Lambda_{2}=2 D_{x}$. We observe that this region extends itself up to a value roughly given by $D_{x}=\lambda_{2, \alpha} / 2$. Once the only difference between the layers $\alpha$ and $\beta$ refers to the adopted numbering, they share the same topology and related properties, like the spectra associated with the possible matrix representations. Therefore, the horizontal lines $\lambda_{2, \alpha}$ and $\lambda_{2, \beta(s)}(\forall s)$ are always coincident whenever we consider multiplexes assembled by layers with identical topology. Two consequences follow from this property: (i) Any multiplex where the layer $\beta$ is obtained from relabeling nodes of layer $\alpha$ is always superdiffusive; (ii) if $\beta(s=0) \neq \alpha$, the horizontal line $\lambda_{2, \beta(s)}$ does not depend on $s$, but superdiffusion may occur depending on $D_{x}$ and on $s$.
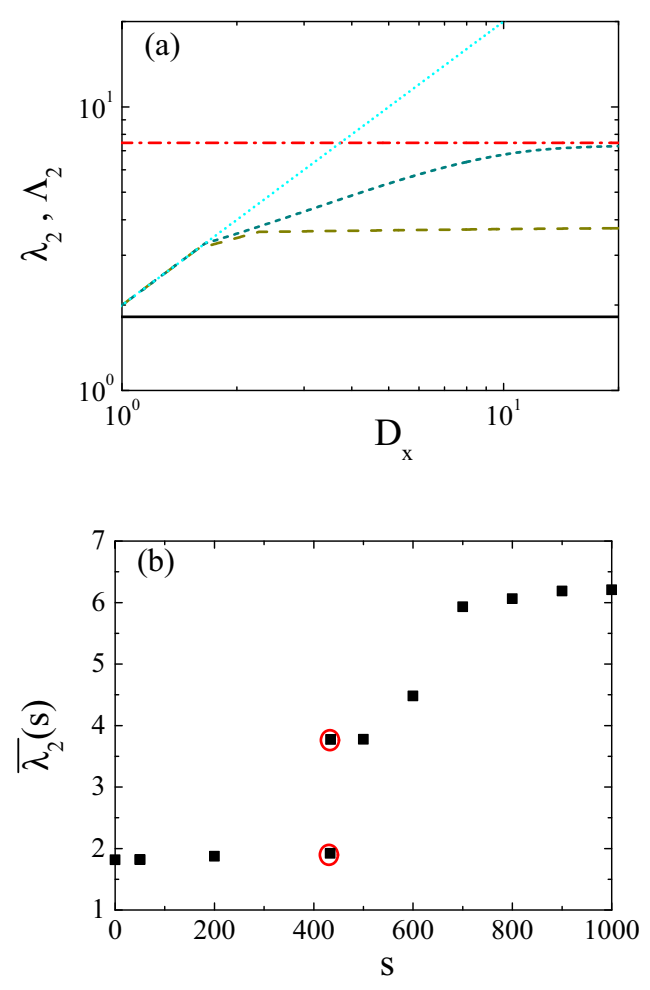

FIG. 4. Typical results produced within the adopted framework for multiplexes assembled from one single $N=500, p=0.01 \mathrm{ER}$ network. (a) Dependency of $\lambda_{2}$ on $D_{x}: \lambda_{2}=2 D_{x}$ (cyan short-dotted line), $\lambda_{2}(\alpha)=\lambda_{2}(\beta)$ (black solid line), $\overline{\lambda_{2}}\left(s=10^{7}\right)$ (red dash-dotted line), $\Lambda_{2}\left(D_{x} ; s=434\right)$ (dark yellow dashed line), $\Lambda_{2}\left(D_{x} ; s=10^{7}\right)$ (dark cyan short-dashed line). (b) Dependency of $\overline{\lambda_{2}}(s)$ on $s$. The red circles around squares indicate the values $s=433$ and 434, where the value of $\overline{\lambda_{2}}(s)$ jumps by a large amount. 

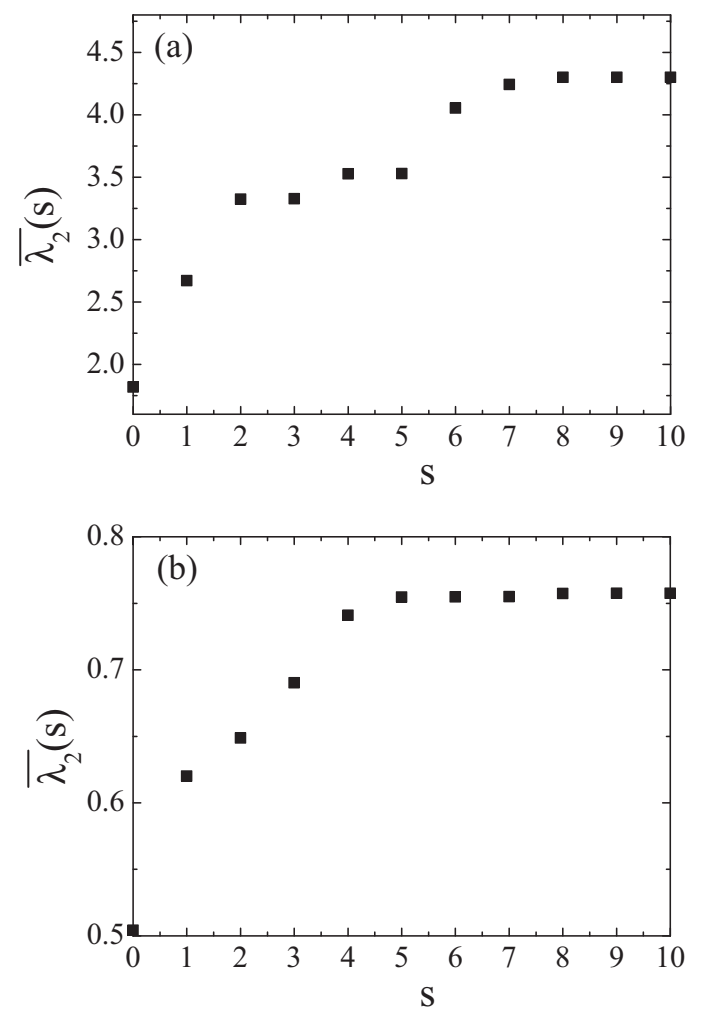

FIG. 5. Dependency of $\overline{\lambda_{2}}(s)$ on $s$ by rewiring targeted pairs of nodes in $\beta$ for an $N=500$ ER multiplex with $p=0.01$ (a), and an $N=750$ ER multiplex with $p=0.008$ (b).

When $D_{x}>\lambda_{2, \alpha} / 2$, we notice that $\Lambda_{2}\left(D_{x} ; s=434\right) \leqslant$ $\Lambda_{2}\left(D_{x} ; s=10^{7}\right) \forall D_{x}$, the same relationship obtained for $\delta(s=434)<\delta\left(s=10^{7}\right)$, as shown in Fig. 3(a). This direct association, which suggests a direct relation between layer dissimilarity and diffusion, is a typical result observed very often in our investigation, especially when the multiplex layers are sparse. We observe that, for multiplexes assembled with ER layers with larger values $p$, e.g., $p=0.1$ and $N=500$, similar features to those described above have also been found.

The dependency of $\bar{\lambda}_{2}$ on $s$, which provides the $D_{x} \rightarrow$ $\infty$ asymptotic behavior of $\Lambda_{2}$, is illustrated in Fig. 4(b). It is characterized by a monotonic increase with respect to $s$, with the presence of some large increments at some specific values of $s$. This is what happens, for instance, when we move from $s=434$ to 435 , indicated by red circles around the black squares. We notice that, from $s=0$ to $433, \bar{\lambda}_{2}(s)$ increases by a very small amount, as compared to a sharp gain of almost $100 \%$ of its value at $s=434$.

The finding that sharp gains in global multiplex diffusion can be obtained by a judicious rewiring of a few nodes in the layer $\beta$ is corroborated by the optimized rewiring strategy described in Sec. III C. This is illustrated in Fig. 5. In panel (a), we show how $\bar{\lambda}_{2}(s)$ changes for the first 10 rewiring steps selected by the optimized rewiring algorithm for the same multiplex used in Fig. 4, and in panel (b) we show similar results for an $N=750 p=0.008$ ER network. In (a), the first detected pair of nodes corresponds to the same pair of nodes exchanged in the $s=434$ step in the random procedure [see
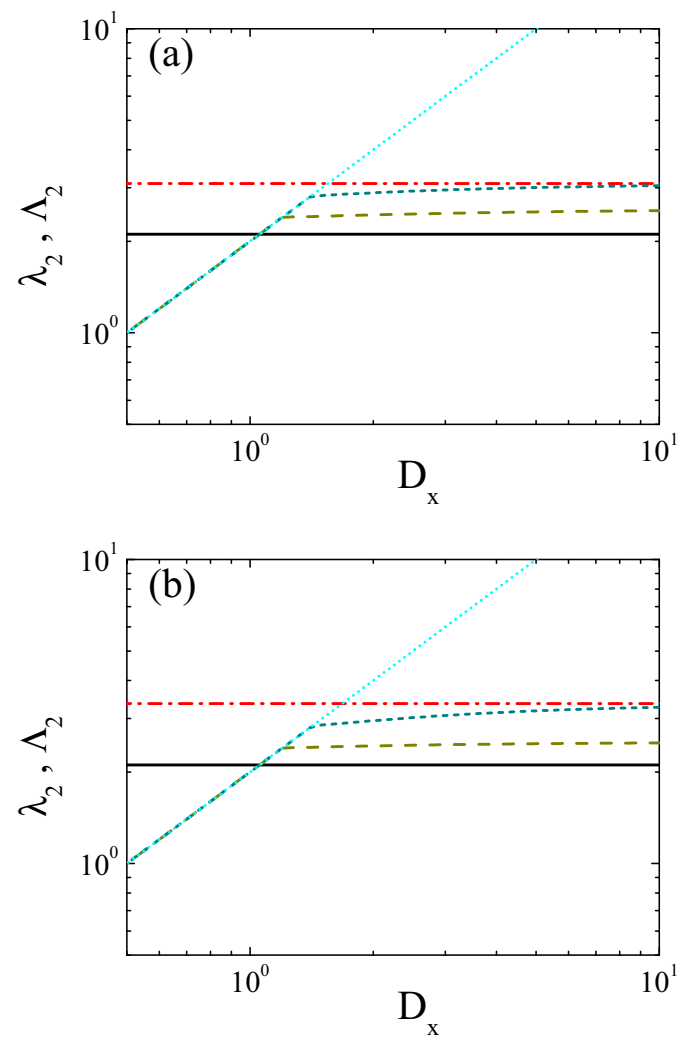

FIG. 6. Typical results produced within the adopted framework for multiplexes assembled from one single $N=1000,\langle k\rangle=4 \mathrm{BA}$ network. (a) Dependency of $\lambda_{2}$ on $D_{x}$ for random rewiring: $\lambda_{2}=2 D_{x}$ (cyan short-dotted line), $\lambda_{2}(\alpha)=\lambda_{2}(\beta)$ (black solid line), $\overline{\lambda_{2}}\left(s=10^{7}\right.$ ) (red dash-dotted line), $\Lambda_{2}\left(D_{x} ; s=500\right)$ (dark yellow dashed line), $\Lambda_{2}\left(D_{x} ; s=10^{7}\right)$ (dark cyan short-dashed line). (b) Dependency of $\lambda_{2}$ on $D_{x}$ for the optimal BA rewiring scheme: $\lambda_{2}=2 D_{x}$ (cyan shortdotted line), $\lambda_{2}(\alpha)=\lambda_{2}(\beta)$ (black solid line), $\overline{\lambda_{2}}(s=499)$ (red dashdotted line), $\Lambda_{2}\left(D_{x} ; s=256\right)$ (dark yellow dashed line), $\Lambda_{2}\left(D_{x} ; s=\right.$ 499) (dark cyan short-dashed line).

Fig. 4(b)]. We notice that this single change in the $\beta$ layer produces an increase of $47 \%$ in the value of $\bar{\lambda}_{2}$, an amazing indication of the effectiveness of this process. We also notice that, after 10 steps, $\bar{\lambda}_{2}(s=10) / \bar{\lambda}_{2}(s=0)=2.37$. In (b), the first exchange causes an increase $\sim 25 \%$ in the value of $\bar{\lambda}_{2}(s)$. Therefore, despite the different values of $\lambda_{2}^{\alpha}$ and $\bar{\lambda}_{2}(s)$ in the two cases, both multiplexes have similar qualitative behavior. This is a clear indication that the rewiring procedure identifies, in a very precise way, which changes in the multiplex structure allow for a very sharp increase of diffusion.

The results obtained for multiplexes where $\alpha=\beta(s=0)$ are BA networks display similar features, as illustrated in Figs. 6(a) and 6(b): the dependency of $\Lambda_{2}$ with respect to $D_{x}$ for several values of $s$ indicates that it increases in a monotonic and uniform way with respect to both control parameters. The dependency of $\overline{\lambda_{2}}(s)$ with respect to $s$ also follows the same overall pattern shown in Fig. 4(b) for the ER multiplexes. However, a difference appears when we apply the optimized rewiring strategy: providing a targeted rewiring $\hat{i} \leftrightarrow \hat{\ell}$ and of the next pairs of nodes identifying that algorithm does not lead to a sharp increase of $\Lambda_{2}$, as observed in Fig. 5 for 

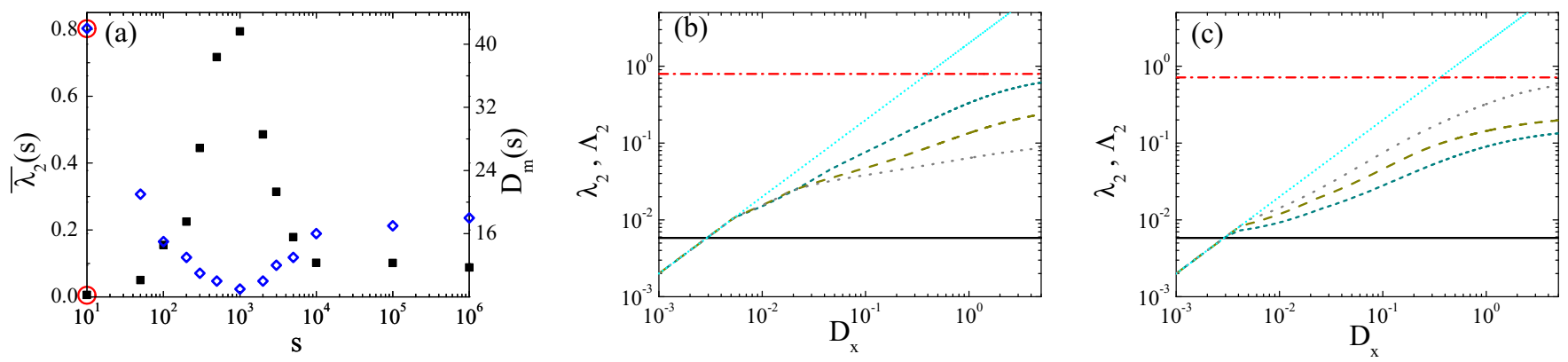

FIG. 7. Typical results produced within the adopted framework for multiplexes assembled from one single $N=500, \rho=0.05$ WS network. (a) Dependency of $\overline{\lambda_{2}}(s)$ (black squares) and $D_{m}(s)$ (blue diamonds) on $s$. The symbols surrounded by red circles on the left vertical axis, which correspond to the values at $s=0$, have been inserted for the purpose of comparison. (b) Dependency of $\lambda_{2}$ on $D_{x}$ for random rewiring: $\lambda_{2}=2 D_{x}$ (cyan short-dotted line), $\lambda_{2}(\alpha)=\lambda_{2}(\beta)$ (black solid line), $\overline{\lambda_{2}}\left(s=10^{3}\right)$ (red dash-dotted line), $\Lambda_{2}\left(D_{x} ; s=10^{3}\right)$ (dark cyan short-dashed line), $\Lambda_{2}\left(D_{x} ; s=3000\right)$ (dark yellow dashed line), $\Lambda_{2}\left(D_{x} ; s=10^{4}\right)$ (gray dots). (c) Dependency of $\lambda_{2}$ on $D_{x}$ for random rewiring: $\lambda_{2}=2 D_{x}$ (cyan short-dotted line), $\lambda_{2}(\alpha)=\lambda_{2}(\beta)$ (black solid line), $\overline{\lambda_{2}}\left(s=10^{3}\right)$ (red dash-dotted line), $\Lambda_{2}\left(D_{x} ; s=100\right)$ (dark cyan short-dashed line), $\Lambda_{2}\left(D_{x} ; s=200\right)$ (dark yellow dashed line), $\Lambda_{2}\left(D_{x} ; s=500\right)$ (gray dots).

the ER assembled multiplexes. On the other hand, a targeted rewiring of pairs of nodes with large and small degree is able to produce a relatively fast increase in $\Lambda_{2}$, as illustrated in the Fig. 6(b).

Our investigation also considered the impact of rewiring $\beta$ on multiplexes based on initially identical layers of a WS small-world network, where a uniform linear chain with connections between nearest and next-nearest neighbors has links randomly rewired with probability $\rho$. We found that, while $\delta$ increases monotonically with $s$ (up to small fluctuations), the same is not observed either for $\Lambda_{2}$ or $\bar{\lambda}_{2}(s)$ when $s$ becomes large enough. This contrasts with the observed behavior for the two former network types. Indeed, Fig. 7(a) illustrates that, for $\rho=0.05, \bar{\lambda}_{2}(s)$ goes through a maximum at $s \sim 1000$, in opposition to the pattern in Fig. 4(b). It was possible to identify that such a behavior is correlated with the dependency of topological features of WS multiplexes with respect to $s$, for instance, the multiplex's shortest path, and the multiplex diameter $D_{m}(s)$. The behavior of $D_{m}(s)$ with respect to $s$ is also illustrated in Fig. 7(a). Note that, for small values of $\rho$, the diameter of WS networks is still much larger when compared to those of the ER counterparts. When the MC procedure starts, $\delta$ increases with $s$ and this also causes a reduction in the value of $D_{m}$. However, after a certain value of $s$ (here $s \sim 10^{3}$ ), the behavior of $D_{m}$ changes and it increases again, irrespective of the fact that $\delta$ still grows with $s$. The results suggest that the diffusive properties of WS multiplexes are strongly related to $D_{m}$. On the other hand, the results for ER and BA multiplexes show that $D_{m}$ remains almost constant, so that $\bar{\lambda}_{2}(s)$ keeps its increasing behavior with $s$ and $\delta(s)$, eventually reaching a stationary situation.

Figure 7(b) shows that, when we consider $\Lambda_{2}$ as a function of $D_{x}$ for successive values of $s$, the corresponding curves cross each other in several points, a feature that was absent in Figs. 4 and 6. The results in Fig. 7(c) correspond to multiplexes obtained for $s \leqslant 1000$, in the range of $s$ where $\bar{\lambda}_{2}(s)$ still increases with $s$. In accordance with the expected limit behavior indicated by Fig. 7(a), for small values of $s$ (typically $s \lesssim 500$ ), the typical behavior shown for ER and BA multiplexes still holds, but these features are lost when $s$ increases beyond this threshold. Finally, we observe that the patterns displayed in Fig. 7 are also observed for other values of $N$ and $\rho$ that lead to typical WS small-world networks.
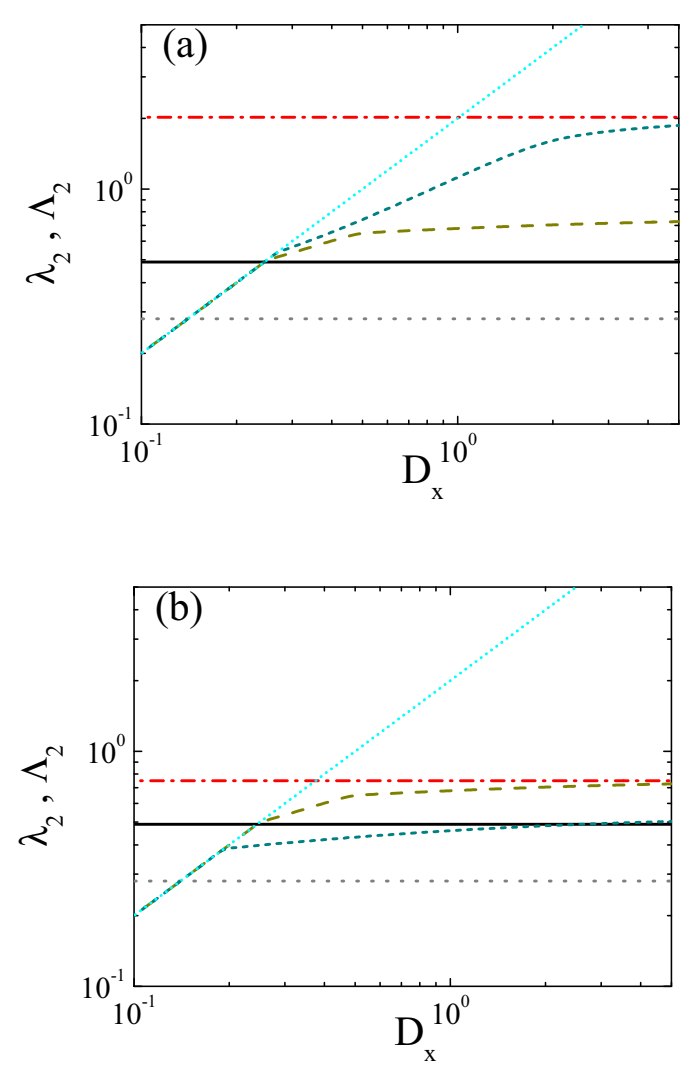

FIG. 8. Typical results for multiplexes assembled by two realizations of $N=500, p=0.01$ ER networks. (a) Dependency of $\lambda_{2}$ on $D_{x}$ for $\delta_{+}$multiplexes: $\lambda_{2}=2 D_{x}$ (cyan short-dotted line), $\lambda_{2}(\alpha)$ (black solid line), $\lambda_{2}(\beta)$ (gray dots), $\overline{\lambda_{2}}\left(s=10^{7}\right.$ ) (red dash-dotted line), $\Lambda_{2}\left(D_{x} ; s=0\right)$ (dark yellow dashed line), $\Lambda_{2}\left(D_{x} ; s=10^{7}\right)$ (dark cyan short-dashed line). (b) Dependency of $\lambda_{2}$ on $D_{x}$ for $\delta_{-}$multiplexes: $\lambda_{2}=2 D_{x}$ (cyan short-dotted line), $\lambda_{2}(\alpha)$ (black solid line), $\lambda_{2}(\beta)$ (gray dots), $\overline{\lambda_{2}}(s=0)$ (red dash-dotted line), $\Lambda_{2}\left(D_{x} ; s=0\right)$ (dark yellow dashed line), $\Lambda_{2}\left(D_{x} ; s=10^{7}\right)$ (dark cyan short-dashed line). 

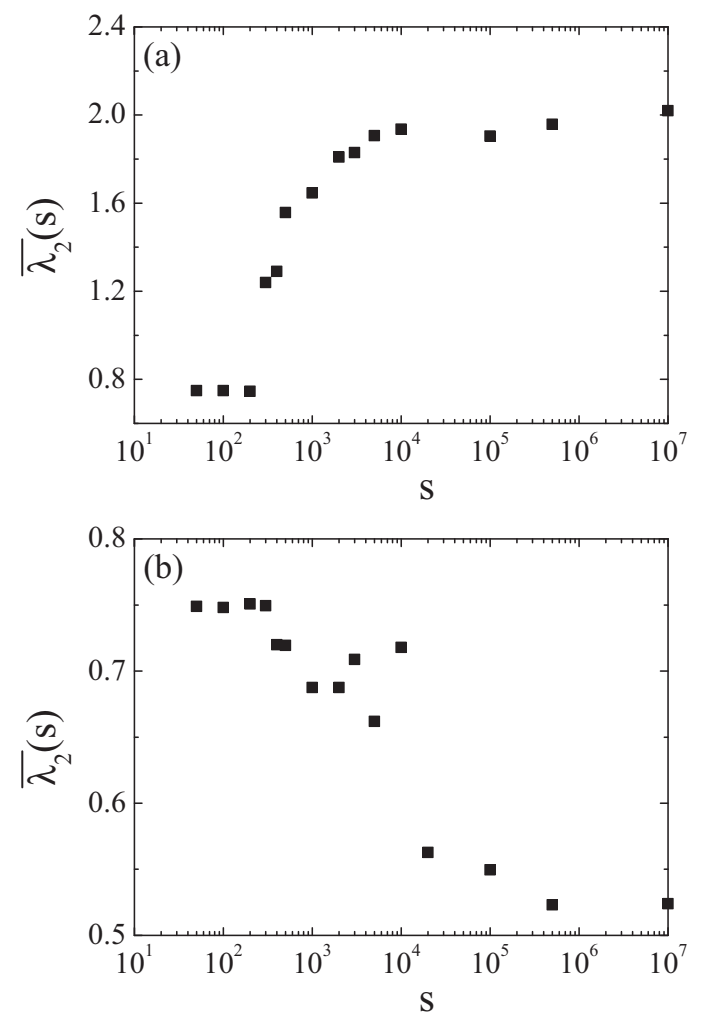

FIG. 9. Dependency of $\overline{\lambda_{2}}(s)$ as a function of $s$ for multiplexes assembled by two realizations of $N=500, p=0.01$ ER networks. Panels (a) and (b) correspond to the situations in which the network distance is given by $\delta_{+}$and $\delta_{-}$, respectively.

When $\rho$ increases, the features illustrated in Fig. 7 become less pronounced.

Now we discuss the relationship between $\delta$ and $\Lambda_{2}$ based on results for multiplexes where $\alpha$ and $\beta(s=0)$ correspond to two different realizations of networks generated by the same procedure. The dependency between $\delta$ and $s$ for ER and BA multiplexes has been presented in Figs. 3(b) and 3(c). Figures 8(a) and 8(b) illustrate the behavior of $\Lambda_{2}$ as a function of $D_{x}$ for two values of $s$ and, respectively, for the two situations described by $\delta_{+}(s)$ and $\delta_{-}(s)$. As expected, two independent realizations of the same algorithm may give rise to networks with different diffusive properties, which is indicated by different values of $\lambda_{2}^{\alpha}$ and $\lambda_{2}^{\beta}$. Finally, note that, if $s_{1}<s_{2}, \Lambda_{2}\left(D_{x} ; s_{1}\right)<\Lambda_{2}\left(D_{x} ; s_{2}\right)\left[\Lambda_{2}\left(D_{x} ; s_{1}\right)>\right.$ $\left.\Lambda_{2}\left(D_{x} ; s_{2}\right)\right]$ for $\delta_{+}\left(\delta_{-}\right)$multiplexes.

The examples in Fig. 8 suggest an overall agreement with the observed behavior for ER multiplexes starting with identical layers, as illustrated in Fig. 9. There we draw the behavior of $\bar{\lambda}_{2}(s)$ as a function of $s$ for the same multiplexes used in Fig. 8. Although the general tendency indicates that $\bar{\lambda}_{2}(s)$ increases (decreases) when $\delta_{+}$increases ( $\delta_{-}$decreases), it is subject to the presence of small-amplitude fluctuations to this rule, especially for the $\delta_{-}$branch. Once similar patterns are obtained for other multiplex realizations with the same parameters, the general conclusion is that a direct relationship between $\Lambda_{2}$ and $\delta$ is valid for sparse ER networks. Our results also show that the magnitude of the deviations from
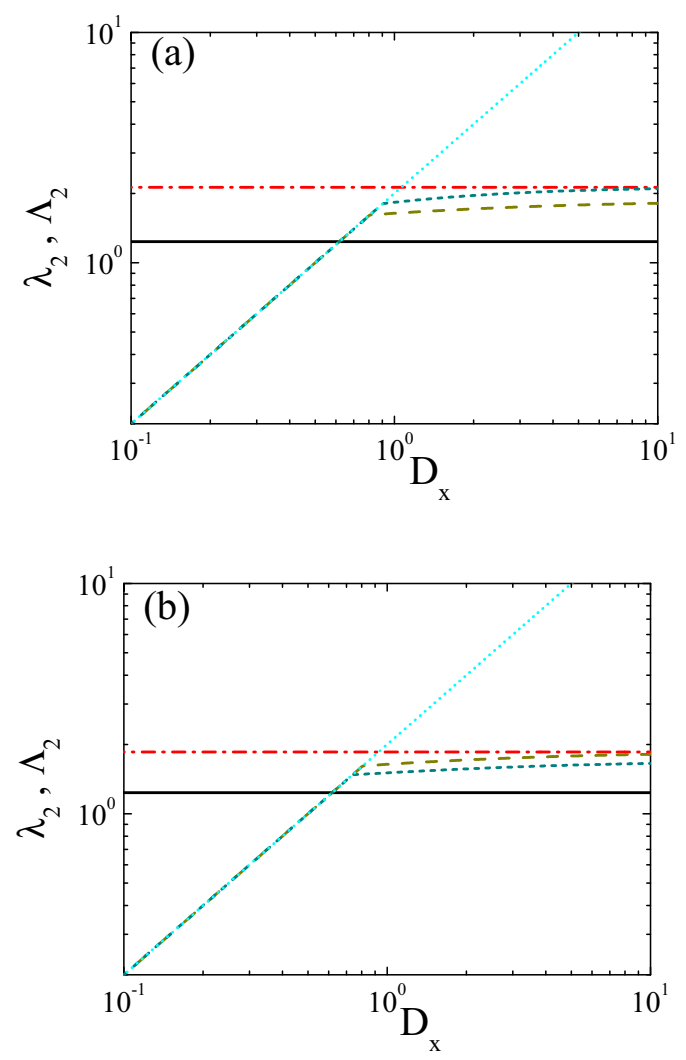

FIG. 10. Typical results for multiplexes assembled by two realizations of $N=1000$, BA networks. (a) Dependency of $\lambda_{2}$ on $D_{x}$ for $\delta_{+}$multiplexes: $\lambda_{2}=2 D_{x}$ (cyan short-dotted line), $\lambda_{2}(\alpha)$ (black solid line), $\overline{\lambda_{2}}\left(s=10^{7}\right)$ (red dash-dotted line), $\Lambda_{2}\left(D_{x} ; s=0\right)$ (dark yellow dashed line), $\Lambda_{2}\left(D_{x} ; s=10^{7}\right)$ (dark cyan short-dashed line). (c) Dependency of $\lambda_{2}$ on $D_{x}$ for $\delta_{-}$multiplexes: $\lambda_{2}=2 D_{x}$ (cyan short-dotted line), $\lambda_{2}(\alpha)$ (black solid line), $\overline{\lambda_{2}}(s=0)$ (red dash-dotted line), $\Lambda_{2}\left(D_{x} ; s=0\right)$ (dark yellow dashed line), $\Lambda_{2}\left(D_{x} ; s=10^{7}\right.$ ) (dark cyan short-dashed line). For the sake of clearer pictures, the horizontal line $\lambda_{2}(\beta)$ is not shown, once it is different but very close to $\lambda_{2}(\alpha)$.

the monotonic behavior of $\bar{\lambda}_{2}(s) \times s$ is of the same order of magnitude even beyond the sparse network regime, e.g., when $p$ increases up to 0.1 when $N=500$.

We also remark that the use of the optimized rewiring procedure when $\alpha \neq \beta(s=0)$ does not produce the same effect observed when $\alpha=\beta(0)$. Although we can use the same procedure to identify $\hat{i}$ and $\hat{\ell}$, the operation $\hat{i} \leftrightarrow \hat{\ell}$ does not lead to a large increase in the values of $\delta$ and $\bar{\lambda}_{2}(s)$ as when the initial layers are identical.

For BA multiplexes, the overall behavior of $\Lambda_{2}$ follows the pattern that displayed sparse ER multiplexes. Figures 10(a) and 10(b) illustrate the behavior of $\Lambda_{2}$ as a function of $D_{x}$ for several values of $s$ and, respectively, for the two situations described by $\delta_{+}(s)$ and $\delta_{-}(s)$ in Fig. 3(c). The general behavior agrees qualitatively with the previously observed tendency in ER multiplexes to have a direct relationship between $\delta$ and $\Lambda_{2}$. Graphs for $\bar{\lambda}_{2}(s) \times s$ are also characterized by an overall monotonic relationship with respect to $\delta$. As in the case of ER multiplexes, small fluctuations may lead, within small $s$ intervals, to deviations from the general tendency. 
With regard to the use of the optimized rewiring, we notice that the procedure based on rewiring pairs of nodes with large and small degree, as used in multiplexes based on identical BA networks, offers again a good strategy to accelerate the MC search process. Indeed, the increase in the value of $\Lambda_{2}$ along with that of $\delta$ occurs at a much faster pace when this choice is used.

The behavior of multiplexes assembled by two distinct realizations of WS networks is not monotonic. As was observed when working with two identical layers at $s=0$, $\bar{\lambda}_{2}(s)$ has the tendency to form a peak when $s$ increases together with $\delta_{+}(s)$, and a minimum when $s$ increases and $\delta_{-}(s)$ decreases. These changes are correlated with the values of $D_{m}(s)$, which shows a minimum (maximum) when we follow the multiplexes generated by $\delta_{+}(s)\left[\delta_{-}(s)\right]$. In accordance with this overall picture, $\Lambda_{2}$ shows a complex behavior with respect to both $s$ and $D_{x}$. The magnitude of these changes may depend on the value of $\rho$ and $N$.

\section{v. CONCLUSIONS}

This work has investigated the relation between diffusion in a multiplex and the differences between the topology of its different layers. We have focused our attention on two-layer multiplexes, so that this relation could be quantified in terms of two well-defined parameters: the multiplex diffusive relaxation time $\tau=1 / \Lambda_{2}$, and the interlayer structural difference $\delta$. To closely follow this dependency, a MC procedure was implemented in such a way that $\delta$ could increase or decrease by selectively rewiring one of the layers in the multiplex $(\beta)$, while the values of $\Lambda_{2}$ were evaluated at suitable values of MC steps $s$. For these values, we followed the dependency of $\Lambda_{2}$ with respect to the interlayer diffusion coefficient $D_{x}$, and we also evaluated $\bar{\lambda}_{2}$, which corresponds to the theoretical asymptotic value $\Lambda_{2}$ when $D_{x} \rightarrow \infty$.
The reported results for three well-known network sets uncovered an overwhelming tendency for a direct relationship between $\Lambda_{2}\left(D_{x} ; s\right)$ and $\delta(s)$. This is valid for two different choices for the layers $\alpha$ and $\beta(s=0)$. When $\alpha=\beta(0), \delta$ necessarily increases with $s$, and so does $\Lambda_{2}$. On the other hand, $\delta$ can be set to increase and decrease when $\alpha \neq \beta(0)$, with a general trend of $\Lambda_{2}$ to behave accordingly. Some exceptions of the general trend were observed when small-world WS networks were subject to a very large number of rewiring steps. Here we could see that, despite a monotonic increase (or decrease) of $\delta, \Lambda_{2}$ moves in the opposite direction, which seems to be correlated with a change in the behavior of the multiplex diameter with respect to $s$.

Although the increase or decrease of $\Lambda_{2}$ proceeds through a series of small increments resulting from rewiring random pairs of $\beta$ links, we were able to identify alternative procedures to speed up the rewiring process of two initially identical layers, in the sense that the values of $\delta$ and $\Lambda_{2}$ can be significantly increased after a very small number of rewired pairs of links. This opens the used framework to applications in real-world situations, in which the diffusion process can be controlled by playing with a small subset of nodes in a multiplex. The extension of the adopted framework to include multiplexes with a larger number of layers is a natural and interesting consequence of this work.

\section{ACKNOWLEDGMENTS}

The partial financial support from $\mathrm{CNPq}$ and CAPES (Brazilian agencies) is acknowledged. J.G.G. acknowledges a CNPQ (401758/2013-3 PVE) grant within the Science without Borders action, the Spanish MINECO under projects FIS201455867-P and FIS2015-71582-C2, and the Departamento de Industria e Innovación del Gobierno de Aragón y Fondo Social Europeo (FENOL group E-19).
[1] S. Boccaletti, G. Bianconi, R. Criado, C. I. Del Genio, J. GómezGardeñes, M. Romance, I. Sendiña-Nadal, Z. Wang, and M. Zanin, Phys. Rep. 544, 1 (2014).

[2] M. Kivelä, A. Arenas, M. Barthelemy, J. P. Gleeson, Y. Moreno, and M. A. Porter, J. Comp. Networks 2, 203 (2014).

[3] J. F. Donges, H. C. Schultz, N. Marwan, Y. Zou, and J. Kurths, Eur. Phys. J. B 84, 635 (2011).

[4] J. Gao, S. V. Buldyrev, H. E. Stanley, and S. Havlin, Nat. Phys. 8, 40 (2012).

[5] F. Battiston, V. Nicosia, and V. Latora, Phys. Rev. E 89, 032804 (2014).

[6] S. V. Buldyrev, R. Parshani, G. Paul, H. E. Stanley, and S. Havlin, Nature (London) 464, 1025 (2010).

[7] F. R. Chung, Spectral Graph Theory, Regional Conference Series in Mathematics Vol. 92 (American Mathematical Society, Providence, RI, 1997).

[8] A. Solé-Ribalta, M. De Domenico, N. E. Kouvaris, A. DíazGuilera, S. Gómez, and A. Arenas, Phys. Rev. E 88, 032807 (2013).

[9] R. Parshani, S. V. Buldyrev, and S. Havlin, Phys. Rev. Lett. 105, 048701 (2010).
[10] L. Solá, M. Romance, R. Criado, J. Flores, A. García del Amo, and S. Boccaletti, Chaos 23, 033131 (2013).

[11] M. De Domenico, A. Solé-Ribalta, E. Cozzo, M. Kivelä, Y. Moreno, M. A. Porter, S. Gómez, and A. Arenas, Phys. Rev. X 3, 041022 (2013).

[12] A. Cardillo, J. Gómez-Gardeñes, M. Zanin, M. Romance, D. Papo, F. Del Pozo, and S. Boccaletti, Sci. Rep. 3, 1344 (2013).

[13] A. Cardillo, M. Zanin, J. Gómez-Gardeñes, M. Romance, A. García del Amo, and S. Bocaletti, Eur. J. Spec. Top. 215, 23 (2013).

[14] M. Szell, R. Lambiotte, and S. Thurner, Proc. Natl. Acad. Sci. (USA) 107, 13636 (2010).

[15] R. Gallotti and M. Barthelemy, Sci. Rep. 4, 6911 (2014).

[16] R. Gallotti, M. Porter, and M. Barthelemy, Sci. Adv. 2, e1500445 (2016).

[17] L. Lotero, R. Hurtado, L. M. Floría, and J. Gómez-Gardeñes, R. Soc. Open Sci. 3, 150654 (2016).

[18] D. Cellai, E. Lopez, J. Zhou, J. P. Gleeson, and G. Bianconi, Phys. Rev. E 88, 052811 (2013).

[19] N. Azimi-Tafreshi, J. Gómez-Gardeñes, and S. N. Dorogovtsev, Phys. Rev. E 90, 032816 (2014). 
[20] G. Bianconi and F. Radicchi, Phys. Rev. E 94, 060301 (2016).

[21] A. Saumell-Mendiola, M. A. Serrano, and M. Boguñá, Phys. Rev. E 86, 026106 (2012).

[22] C. Granell, S. Gómez, and A. Arenas, Phys. Rev. Lett. 111, 128701 (2013).

[23] E. Cozzo, R. A. Baños, S. Meloni, and Y. Moreno, Phys. Rev. E 88, 050801 (2013).

[24] C. Buono, L. Alvarez-Zuzek, P. Macri, and L. Braunstein, PLoS ONE 9, e92200 (2014).

[25] F. Darabi Sahneh and C. Scoglio, Phys. Rev. E 89, 062817 (2014).

[26] J. P. Gleeson, J. A. Ward, K. P. O’Sullivan, and W. T. Lee, Phys. Rev. Lett. 112, 048701 (2014).

[27] F. Radicchi and A. Arenas, Nat. Phys. 9, 717 (2013).

[28] J. Gómez-Gardeñes, M. De Domenico, G. Gutiérrez, A. Arenas, and S. Gómez, Philos. Trans. R. Soc. London, Ser. A 373, 20150117 (2015).

[29] J. Gómez-Gardeñes, I. Reinares, A. Arenas, and L. M. Floría, Sci. Rep. 2, 620 (2012).

[30] J. T. Matamalas, J. Poncela-Casasnovas, and A. Arenas, Sci. Rep. 5, 9519 (2015).

[31] Z. Wang, A. Szolnoki, and M. Perc, Europhys. Lett. 97, 48001 (2012).

[32] Z. Wang, L. Wang, and M. Perc, Phys. Rev. E 89, 052813 (2014).

[33] F. Sorrentino, New J. Phys. 14, 033035 (2012).

[34] L. V. Gambuzza, M. Frasca, and J. Gómez-Gardeñes, Europhys. Lett. 110, 20010 (2015).

[35] R. Sevilla-Escoboza, R. Gutiérrez, G. Huerta-Cuellar, S. Boccaletti, J. Gómez-Gardeñes, A. Arenas, and J. M. Buldú, Phys. Rev. E 92, 032804 (2015).

[36] C. I. Del Genio, J. Gómez-Gardeñes, I. Bonamassa, and S. Boccaletti, Sci. Adv. 2, e1601679 (2016).
[37] N. Kouvaris, S. Hata, and A. Díaz-Guilera, Sci. Rep. 5, 10840 (2015).

[38] M. De Domenico, C. Granell, M. A. Porter, and A. Arenas, Nat. Phys. 12, 901 (2016).

[39] S. Gomez, A. Diaz-Guilera, J. Gomez-Gardeñes, C. J. PerezVicente, Y. Moreno, and A. Arenas, Phys. Rev. Lett. 110, 028701 (2013).

[40] J. A. Almendral and A. Díaz-Guilera, New J. Phys. 9, 187 (2007).

[41] R. F. Andrade, J. G. Miranda, S. T. Pinho, and T. P. Lobão, Phys. Lett. A 372, 5265 (2008).

[42] M. E. Newman, J. Stat. Phys. 101, 819 (2000).

[43] R. Albert and A.-L. Barabási, Rev. Mod. Phys. 74, 47 (2002).

[44] S. Boccaletti, V. Latora, Y. Moreno, M. Chavez, and D.-U. Hwang, Phys. Rep. 424, 175 (2006).

[45] M. Newman, Networks: An Introduction (Oxford University Press, Oxford, 2010).

[46] S. N. Dorogovtsev and J. F. Mendes, Evolution of Networks: From Biological Nets to the Internet and WWW (Oxford University Press, Oxford, 2013).

[47] P. Erdös and A. Rényi, Publ. Math. Debrecen 6, 290 (1959).

[48] P. Erdös and A. Rényi, Publ. Math. Inst. Hung. Acad. Sci. 5, 17 (1960).

[49] A.-L. Barabási and R. Albert, Science 286, 509 (1999).

[50] D. J. Watts and S. H. Strogatz, Nature (London) 393, 440 (1998).

[51] V. Latora and M. Marchiori, Eur. Phys. J. B 32, 249 (2003).

[52] R. F. S. Andrade, J. G. V. Miranda, and T. P. Lobão, Phys. Rev. E 73, 046101 (2006).

[53] D. P. Landau and K. Binder, A Guide to Monte Carlo Simulations in Statistical Physics (Cambridge University Press, Cambridge, 2014). 